\title{
Tsafon
}

Revue d'études juives du Nord

$82 \mid 2021$

Enjeux esthétiques dans la littérature après Auschwitz

\section{Le chantier de Champaubert-aux-Bois}

Travail de bûcheronnage : un leurre pour dix-huit Juifs de Lille

\section{Danielle Delmaire}

\section{OpenEdition}

Journals

Édition électronique

URL : https://journals.openedition.org/tsafon/4422

DOI : $10.4000 /$ tsafon.4422

ISSN : 2609-6420

Éditeur

Association Jean-Marie Delmaire

Édition imprimée

Date de publication : 1 décembre 2021

Pagination : 109-132

ISSN : $1149-6630$

Référence électronique

Danielle Delmaire, "Le chantier de Champaubert-aux-Bois », Tsafon [En ligne], 82 | 2021, mis en ligne le 01 décembre 2021, consulté le 12 février 2022. URL : http://journals.openedition.org/tsafon/4422 ; DOI : https://doi.org/10.4000/tsafon.4422 


\title{
Le chantier de Champaubert-aux-Bois
}

\author{
Travail de bûcheronnage : un leurre \\ pour dix-huit Juifs de Lille
}

Danielle Delmaire*

\begin{abstract}
Durant l'année 1942, des dizaines d'hommes juifs sont affectés, comme bûcherons ou manœuvres, à un chantier forestier sur la commune de Champaubert-aux-Bois, sous l'égide de la délégation générale à l'équipement national. La plupart de ces hommes, expédiés sur ce chantier par un bureau de placement, sont originaires de Lille (AD Marne, $1 \mathrm{~W}$ M3100). ${ }^{1}$
\end{abstract}

Ce sont ces deux phrases du livre d'Alexandre Doulut et Lucien Lazare qui ont retenu toute mon attention. Suite à une conversation téléphonique, Alexandre Doulut a eu l'amabilité de me communiquer les fiches qui rendent compte de l'arrivée et du non-retour des Juifs travaillant sur le chantier de Champaubert-aux-Bois (Marne). Qu'il en soit grandement remercié.

Ces fiches sont rédigées par un surveillant du chantier qui note scrupuleusement tout ce qui concerne ces Juifs que l'on peut considérer comme évadés du chantier: détails sur la naissance, l'origine géographique, le domicile et surtout les circonstances du non-retour car ces travailleurs pouvaient sortir du chantier. Elles se trouvent aux Archives départementales de la Marne (AD 51) à la cote indiquée par A. Doulut.

\footnotetext{
* Université de Lille.

${ }^{1}$ Doulut Alexandre \& Lazare Lucien, Ni héros, ni salauds. La population a-t-elle protégé les juifs en France occupée? Lormont, éd. Le Bord de 1'Eau, 2019, p. 160.
} 
À partir de ces documents d'archives des AD 51, cotés 1 W M3100, j'ai pu élaborer deux tableaux qui rendent compte des renseignements sur les travailleurs et sur leur séjour au chantier de Champaubert-aux-Bois.

\section{Tableau 1}

Liste des dix-huit Juifs de Lille ayant travaillé sur le chantier de Champaubert-aux-Bois à l'été 1942

\begin{tabular}{|l|l|l|l|}
\hline NOM Prénom & Date naiss. & Lieu naissance & $\begin{array}{l}\text { Adresse à Lille (sauf } \\
\text { une à Ronchin) }\end{array}$ \\
\hline BLANK Chuya & $20-02-1895$ & Zgiez Pol. & 133 rue Gustave Delory \\
\hline BLIBAUM Lajbus & $08-07-1909$ & Tomaszayg Pol. & 2 rue Doudin \\
\hline BOCHNER Mendel & $15-06-1901$ & Zadlona Pol. & 35 rue Lottin \\
\hline BORNSTEIN Wolf & $20-05-1898$ & Godz Pol. & 38 rue Léonard Danel \\
\hline EPSZTAIN Moczeck & $03-04-1907$ & Szarhe Pol. & 133 rue Gustave Delory \\
\hline GRUDKI Abraham & $05-03-1898$ & $\begin{array}{l}\text { Grod Shohaux } \\
\text { Pol. }\end{array}$ & 73 rue de la Monnaie \\
\hline KURCBARD Mendel & $12-09-1891$ & Rousk Pol. & 49 rue de Tournai \\
\hline LAUFER David & $19-04-1905$ & Tarnobryge Pol. & 136 bd Victor Hugo \\
\hline LIBERMAN Jech & $20-05-1897$ & Kanszyce Pol. & $\begin{array}{l}300 \text { av. Jean Jaurès } \\
\text { Ronchin }\end{array}$ \\
\hline MAJER Joseph & $01-02-1924$ & Sanok Pol. & 18 rue des Tanneurs \\
\hline MAJER Mendel & $10-10-1891$ & Sanok Pol. & 18 rue des Tanneurs \\
\hline MUZNER Pinkas & $03-06-1897$ & Czudec Pol. & 15 rue Frédéric Molley \\
\hline POZNANSKI Azoel & $06-09-1896$ & $\begin{array}{l}\text { Ruda Guzowska } \\
\text { Pol. }\end{array}$ & rue de Poids \\
\hline RAPOPORT Jacques & $19-01-1899$ & Medzin Pol. & 6 rue Saint-Sauveur \\
\hline ROCHMAN Jacob & $30-04-1904$ & Varsovie Pol. & 4 rue des Augustins \\
\hline STULZAFT Bérile & $06-04-1896$ & Vilva Pol. & 49 rue de Tournai \\
\hline WILDER Louis & $04-12-1924$ & Lille France & 73 rue de la Monnaie \\
\hline ZYLBERBERG Lajbus & $07-08-1899$ & Chuiswy Pol. & 11 rue d'Amiens \\
\hline
\end{tabular}


Tableau 2

Arrivées et départs des dix-huit Juifs de Lille ayant travaillé sur le chantier de Champaubert-aux-Bois à l'été 1942

$$
(\mathrm{sd}=\text { sans date })
$$

Sur 18 fiches au total, 17 datent l'arrivée du travailleur et 15 datent le départ. Les départs sont soit constatés à la date indiquée dans le rapport, soit signalés à la date du rapport, le départ a donc pu avoir lieu un peu plus tôt.

\begin{tabular}{|l|l|l|}
\hline NOM Prénom & Arrivée & Départ \\
\hline BLANK Chuya & $13-08$ & sd \\
\hline BLIBAUM Lajbus & $13-08$ & $13-10$ \\
\hline BOCHNER Mendel & $13-08$ & $05-12$ (constaté) \\
\hline BORNSTEIN Wolf & $13-08$ & $12-10$ \\
\hline EPSZTAIN Moczeck & $13-08$ & $08-12$ \\
\hline GRUDKI Abraham & $13-08$ & $10-10$ \\
\hline KURCBARD Mendel & $13-08$ & $10-10$ \\
\hline LAUFER David & $04-09$ & $05-12$ (constaté) \\
\hline LIBERMAN Jech & sd & $21-11$ (constaté) \\
\hline MAJER Joseph & $13-08$ & sd \\
\hline MAJER Mendel & $13-08$ & $24-12$ \\
\hline MUZNER Pinkas & $24-09$ ou 24-10 & $10-12$ \\
\hline POZNANSKI Azoel & $18-05$ ou 13-08 & $05-12$ \\
\hline RAPOPORT Jacques & $13-08$ & $10-10$ \\
\hline ROCHMAN Jacob & $28-08$ & $13-10$ \\
\hline STULZAFT Bérile & $28-08$ & $13-10$ \\
\hline WILDER Louis & $03-05$ & $16-10$ \\
\hline ZYLBERBERG Lajbus & $13-08$ & $\mathrm{sd}$ \\
\hline & &
\end{tabular}




\section{Remarques}

Cette source peut être confrontée à un recensement, effectué probablement à la fin de 1942, qui se trouve dans les archives de l'UGIF ${ }^{2}$ et qui donne d'autres renseignements sur ces travailleurs dont les noms figurent tous dans ce recensement, notamment la composition de la famille ${ }^{3}$. Parfois, l'orthographe du nom varie légèrement, selon la source.

\section{BLIBAUM ou BILBAUER.}

GRUDKI Abraham est le père de Renée STRAUSS née Grudki, témoignage de sa fille qui confirme le travail de son père sur un chantier, à lire en fin d'article.

\section{LAUFER ou LANFER.}

LIBERMAN Jech, l'adresse est indiquée à Lille où il n'y a pas de rue Jean Jaurès, le recensement de l'UGIF donne la même adresse mais à Ronchin dans la proche banlieue de Lille.

MUZNER ou MURNET, la rue Frédéric Molley n'existe pas, c'est probablement la rue Frédéric Mottez où habitent d'autres familles juives. WILDER ou WILNER.

Plusieurs de ces hommes sont domiciliés au même endroit :

133 rue Gustave Delory : BLANK Chuya et EPSZTAIN Moczeck 49 rue de Tournai : KURCBARD Mendel et STULZAFT Bérile 73 rue de la Monnaie : GRUDKI Abraham et WILDER Louis (oncle et neveu : témoignage de Mme Renée Strauss, née Grudki)

18 rue des Tanneurs : MAJER Mendel et MAJER Joseph (il s'agit du père et du fils selon le recensement de l'UGIF)

\footnotetext{
${ }^{2}$ Union Générale des Israélites de France : organisme créé par le gouvernement de Vichy obéissant à une demande allemande. Toutes les associations juives doivent être dissoutes pour intégrer l'UGIF dont le rôle était de représenter tous les Juifs auprès des pouvoirs publics et de leur porter assistance. En réalité, les établissements gérés par l'UGIF furent très souvent des souricières à partir desquelles nombre de Juifs, notamment des enfants, furent déportés.

${ }^{3}$ Le recensement se trouve aux Archives of the YIVO Institute for Jewish Research à New-York: UGIF Archives VI 37-49-52, Census of Nord and Pas-de-Calais. Il est étonnant qu'un recensement des Juifs des départements du Nord et du Pas-de-Calais se trouve dans les archives de l'UGIF qui n'avait pas de représentation dans la zone rattachée au commandement militaire de Bruxelles. Ceci prouverait encore la situation particulière des deux départements placés entre les deux autorités administratives. Sur ce sujet lire Monique Heddebaut, "La spécificité de la Zone dite rattachée à Bruxelles », Tsafon, revue d'études juives du Nord, $\mathrm{n}^{\circ} 79$, printemps-été 2020, p. 17-38.
} 
La plupart ont entre 33 et 51 ans sauf deux jeunes gens MAJER Joseph qui a 18 ans et WILDER Louis qui n'a pas encore 18 ans. Tous sont nés en Pologne sauf le plus jeune, WILDER Louis qui est né à Lille.

\section{Le chantier de Champaubert-aux-Bois}

Le petit village de Champaubert-aux-Bois est connu dès le MoyenÂge, sa population reste modeste durant les $\mathrm{XIX}^{\mathrm{e}}$ et $\mathrm{XX}^{\mathrm{e}}$ siècles, oscillant entre 250 et 350 habitants. Le $1^{\mathrm{er}}$ janvier 1971 , cette commune est rattachée à celle de Giffaumont pour n'en former qu'une seule, celle de GiffaumontChampaubert. La réalisation d'un lac-réservoir a englouti l'ancien village de Champaubert dont il ne reste que l'église sur une presqu'île du lac ${ }^{4}$.

Durant la guerre un « camp forestier » est ouvert, sur la commune, pour exploiter les forêts :

Le camp forestier $n^{\circ} 1854$ de Champaubert-aux-Bois a employé comme bûcherons des juifs recrutés, avec autorisation des autorités occupantes, par le Commissariat à la lutte contre le chômage. Créé en octobre 1940 par le gouvernement de Vichy, cet organisme rattaché au Secrétariat d'état au travail disposait de délégués régionaux dont un pour la région de Champagne, dite aussi région de Châlons qui regroupait l'Aube, la Marne et la Haute-Marne. ${ }^{5}$

La mise en place de ce chantier et l'utilisation d'une main d'œuvre juive recrutée à Paris ${ }^{6}$ et à Lille, dans la zone rattachée au commandement militaire de Bruxelles, met en évidence le caractère hybride et la particularité de l'administration qui gérait les deux départements septentrionaux de la France. Bien que dépendant de Bruxelles, ces deux

\footnotetext{
${ }^{4}$ Selon la description de la commune de Champaubert-aux-Bois sur le site Wikipedia, consulté le 2 novembre 2020.

${ }^{5}$ Jocelyne et Jean-Pierre Husson, Les juifs du camp forestier de Champaubert-aux-Bois pendant la Seconde Guerre mondiale, dossier mis en ligne, sur le site du Centre Régional de Documentation Pédagogique de Reims, http://www.cndp.fr/crdpreims/memoire/enseigner/memoire deportation/shoah51/champaubert.htm, consulté le 2 novembre 2020. Les auteurs signalent la présence de neuf Juifs de Lille, seulement, pour l'été 1942 alors que les documents d'archives AD 51 1W M3100 concernent 18 Juifs de Lille.

${ }^{6}$ Notamment, le père de Violette Jacquet, née Silberstein, «a fait un court séjour » à Champaubert-aux-Bois avant de rejoindre sa famille à Paris pour ensuite se réfugier à Lille. Lettre que Violette Jacquet m'a écrite le 15 février 2005. Lire aussi son témoignage : Les sanglots longs des violons de la mort, Paris, Oskarson et Oslo éditions, 2005. Violette Jacquet fut déportée à Auschwitz dont elle revint parce qu'elle put être intégrée à l'orchestre de femmes pour lesquelles les conditions de vie étaient moins rudes.
} 
départements abritaient un bureau de recrutement pour un travail effectué en dehors de cette zone rattachée ${ }^{7}$.

Les gendarmes signataires des fiches renseignant sur le non-retour des « israélites » précisent que ceux-ci « sont envoyés par le commissariat au chantier (Délégation Générale à l'équipement), avec l'autorisation des autorités occupantes $»$.

Sans ressources, à cause de l'interdiction de travailler ou de la spoliation de leurs biens, des Juifs de Lille se sont rendus à un bureau de placement (A. Doulut et L. Lazare) qui les a dirigés vers le «camp forestier» de Champaubert-aux-Bois. Les Juifs qui y sont employés peuvent obtenir des permissions. C'est au cours de ces permissions que les hommes ne rentrent pas et leur disparition est signalée par des gendarmes qui recueillent la déclaration du chef de chantier ayant constaté le nonretour des hommes. Les fiches sont transmises à la préfecture de la Marne. Les Lillois ne sont donc pas les seuls à s'être présentés pour travailler sur ce chantier mais je n'ai retenu que les fiches les concernant, soit 18.

Les conditions d'enrôlement des travailleurs et celles de leur détention avec possibilité d'obtenir des permissions sont aussi celles des travailleurs agricoles des WOL dans les Ardennes ${ }^{8}$, encore que ces derniers étaient souvent accompagnés de leur famille, ou des internés au camp des Groupements des Travailleurs Étrangers (GTE) de Troyes ${ }^{9}$.

\footnotetext{
${ }^{7}$ Cf. Monique Heddebaut, « La spécificité de la Zone dite rattachée à Bruxelles », Tsafon, revue d'études juives $d u$ Nord, article cité, p. 17-38.

${ }^{8}$ WOL : WirtschaftOberLeitung, assurait la mise en culture de terres au profit de l'Allemagne, c'était une filiale de l'Ostland, entreprise privée bien en vue à Berlin. Elle employait des travailleurs juifs mais aussi des prisonniers qui devaient cultiver des terres dont les propriétaires avaient été dépossédés. Sur les travailleurs juifs dans les fermes ardennaises de la WOL, on peut se reporter, entre autres, à une étude à charge contre l'UGIF de Maurice Rajsfus, Des juifs dans la collaboration, l'U.G.I.F. 1941-1944, Paris, études et documentation internationales, 1980, p. 209-230. Idem, Des juifs dans la collaboration (II). Une terre promise? (1941-1944), Paris, L'Harmattan, 1989, p. 85159 ; ainsi qu'à Christine Dollard-Leplomb, Sauveteurs d'étoiles en Ardennes, Charleville-Mézières, éd. Terres Ardennaises, 2006, dont l'ouvrage repose essentiellement sur des témoignages.

${ }^{9}$ La bibliographie sur le camp de Groupements des Travailleurs Étrangers en tant que tel est limitée, les études se perdent dans des ouvrages qui évoquent plus largement les camps en France. Toutefois, l'on peut tirer quelques renseignements sur le site de l'AJPN (Anonymes, Justes et Persécutés durant la Période Nazie) dans sa page sur le camp JulesFerry, du nom de l'école où furent internés les travailleurs juifs. On peut aussi se reporter à Henri Cahen, « 1940-1944 : les années tragiques de la barbarie nazie », Troyes et ses juifs, Jérusalem, 2001, p. 117-134. Plus précisément encore, il faut consulter la thèse de Rudy Rigaut, "JUIFS » dans la zone littorale du Nord et du Pas-de-Calais, du début du $X I X^{e}$ siècle à la fin des années 2010, co-dirigée par Claire Zalc et Michel-Pierre Chélini, soutenue le 8 juin 2020, université d'Artois, les chapitres 4 et 5 et particulièrement les
} 
Les Juifs enrôlés au chantier de Champaubert-aux-Bois étaient employés aux travaux de bûcheronnage dans les forêts à l'entour du village. Selon l'une des fiches, Blank était «chef de manœuvre à l'heure », Epsztain, Laufer, Majer Mendel et Joseph, Poznanski et Zylberberg étaient « manœuvres à l'heure », tandis que Bochner était « bûcheron à la tâche ». Selon une autre fiche, ils sont bûcherons; ce qui laisse supposer un éventuel salaire ou la promesse d'être payés. Il est arrivé que ces hommes recrutés sur des chantiers de l'Organisation Todt soient désignés comme salariés mais ils ne touchaient quasiment rien, sinon rien du tout. Le plus souvent, ces Juifs avaient été encouragés à se présenter au recrutement sur la promesse d'obtenir un salaire leur permettant de nourrir leur famille et de bénéficier d'une protection évitant l'arrestation.

Quelques temps après son installation dans le Nord, l'ennemi s'était préoccupé, de recruter du personnel qui devait travailler pour la machine de guerre allemande [...] Aux Juifs il s'adresse avec cette fourberie, cette mauvaise foi qui caractérisent l'Allemand. "JUIFS, leur dit-il, vous n'aurez rien à craindre si vous allez travailler dans les entreprises forestières qui vous attendent et vous serez bien traités. Mieux encore, vos femmes, vos enfants resteront sous notre surveillance et nous les protègerons ». Des israélites se laissèrent prendre à cette doucereuse invite qui n'était autre qu'un piège comme les événements allaient le démontrer. Ouvriers, artisans, petits commerçants s'inscrivirent uniquement guidés par l'espoir de sauver femmes et enfants. Ce furent ces familles qui le 11 septembre furent les premières arrêtées. ${ }^{10}$

\section{Le séjour des travailleurs}

Un groupe de onze ou douze hommes (une arrivée n'est pas bien définie) est arrivé le 13 août 1942, les cinq autres, dont l'arrivée est connue, ont été affectés séparément durant l'été 1942.

Les non-retours sont plus échelonnés entre le 10 octobre et le 24 décembre 1942. Soit 15 non-retours en deux mois et demi, la surveillance du chantier semble donc peu sévère et de ce fait on peut s'en échapper :

p. 288 à 324. Les Juifs du Boulonnais furent arrêtés et transférés au GTE de Troyes le 17 décembre 1940, où ils constituèrent des proies faciles à déporter. Rudy Rigaut décrit leur transfert et leur sort à Troyes.

${ }^{10}$ Rapport, non daté mais établi immédiatement après la guerre par le grand rabbin américain Brandris, se trouvant à Lille lors de la Libération et encadrant temporairement les communautés juives du Nord. Archives privées d'Edgard Leser. Le 11 septembre 1942 eut lieu une grande rafle qui décima les communautés juives du Nord et du Pas-deCalais et dont furent victimes des membres appartenant aux familles des hommes de Champaubert-aux-Bois. 
« Je ne suis astreint à aucune surveillance spéciale ; seules les prescriptions relatives aux Israélites m'étaient appliquées. Je ne devais pas quitter mon baraquement après 20 heures, ni sortir du périmètre de mon chantier $»^{11}$. Huit non-retours sont effectués ou constatés entre le 10 octobre et le 16 octobre 1942 et six autres le sont entre le 5 décembre et le 24 décembre 1942, un autre non-retour est constaté le 21 novembre.

15 fiches individuelles rendent compte de ces non-retours et indiquent le nombre d' '« israélites [qui] sont employés au Chantier 1854, à Champaubert-aux-Bois ». Huit d'entre elles sont rédigées le 27 octobre, à cette date 50 « israélites » sont sur le chantier, selon une fiche datée du 3 décembre ils ne sont plus que 32 et selon six fiches datées entre le 12 décembre 1942 et le 8 janvier 1943, ils sont encore moins nombreux : 26. Le chantier perd donc la moitié de son effectif durant les trois derniers mois de l'année. La dureté des conditions de travail et de vie dissuada rapidement ces bûcherons improvisés !

En conséquence, les travailleurs restent peu de temps sur le chantier. Au bout de quelques mois, ils ne rentrent pas de permission et la plupart disparaissent dans la nature. Les rapports signalant leur non-retour concluent toujours à une annonce de recherche au domicile du travailleur mais rien n'est écrit sur le résultat d'une telle enquête.

Ce sont Majer Mendel, Poznanski Azoel et Wilder Louis qui sont restés le plus longtemps sur le chantier : cinq à six mois. Les autres hommes ont quitté le chantier au bout de deux à quatre mois, sans doute vite conscients que le travail était trop pénible pour des avantages incertains. D'après Mme Renée Strauss, son père s'est vite rendu compte que la promesse d'éviter une déportation à sa famille était fallacieuse et la rémunération peu en rapport avec le travail épuisant de bûcheron. Il avait accepté l'offre d'un bureau de placement à Lille qui promettait un salaire et pas de déportation. Se trouvant sans ressources, en 1942, il avait décidé d'aller travailler sur ce chantier (lire le témoignage de Mme Renée Strauss, née Grudki).

À l'été 1942, d'autres camps sont ouverts pour faire travailler des Juifs, sans ressources du fait de la confiscation de leur commerce ou de leur entreprise, notamment pour la construction du Mur de l'Atlantique. Dans le camp de Dannes-Camiers, près de Boulogne-sur-Mer, des Juifs de Belgique durent travailler dans des conditions inhumaines. De même dans

\footnotetext{
${ }^{11}$ Déclaration de Chaïm Bursztyn consignée dans un rapport de la gendarmerie de Châlons-sur-Marne du 20 février 1943, citée dans Jocelyne et Jean-Pierre Husson, Les juifs du camp forestier..., op. cit.
} 
le Judenlager des Mazures (département des Ardennes), des Juifs d'Anvers furent également employés à la fabrication de charbon de bois. Dans les deux cas, ces travailleurs furent déportés à Auschwitz en octobre 1942. La durée de leur internement coïncide avec celle des «bûcherons » de Champaubert-aux-Bois ${ }^{12}$.

\section{Qui étaient ces hommes et leur famille ? Que sont-ils devenus?}

Après avoir décidé de ne pas rentrer au chantier, que sont devenus ces hommes ? Sont-ils retournés à leur domicile comme le suggèrent les rapports? Les archives de la Marne semblent muettes et il faudrait chercher en d'autres fonds, à l'aveuglette en l'absence de toute indication de leur destination après leur sortie du chantier.

Toutefois, les Mémoriaux de Serge Klarsfeld et de Maxime Steinberg, respectivement de la déportation des Juifs de France et de la déportation des Juifs de Belgique ${ }^{13}$ livrent quelques renseignements sur le sort de trois familles et sur celui d'un des bûcherons de Champaubert-auxBois. À la fin des années 1980, j'ai pu consulter d'autres listes aux archives du ministère de la Santé publique et de la Famille à Bruxelles, devenu depuis le Service des Archives des Victimes de la Guerre, toujours sis square de l'Aviation à Bruxelles. Non cotées (à l'époque), il s'agit d'une «Liste alphabétique des 748 Israélites de nationalité française ou nés en France ou domiciliés en France ou arrêtés en France qui ont été internés ou déportés du camp de rassemblement de Malines vers les camps de Haute Silésie » et de la «Liste alphabétique des Israélites arrêtés dans le nord de la France, vraisemblablement y domiciliés, transférés du Nord de la France, en train, au camp de rassemblement de Malines où ils sont arrivés le 12-9-1942. Ils y ont été réunis avec d'autres Israélites domiciliés en Belgique et internés au camp de rassemblement de Malines pour former

\footnotetext{
${ }^{12}$ Sur les camps du Boulonnais, lire Danielle Delmaire, «'Les camps de juifs' dans le nord de la France (1942-1944) », Bulletin de MEMOR, n 8, décembre 1987, p. 47 à 65 et la thèse de Rudy Rigaut, «JUIFS» dans la zone littorale du Nord... op.cit., p. 402409. Sur le Judenlager des Mazures, lire Jean-Émile Andreux, « Mémorial des déportés du Judenlager des Mazures », Tsafon, revue d'études juives du Nord, hors-série ${ }^{\circ} 3$, octobre 2007.

${ }^{13}$ Serge Klarsfeld, Le Mémorial de la Déportation des Juifs de France, Paris, édité et publié par Beate et Serge Klarsfeld, 1978, depuis 2012 l'ouvrage est consultable en ligne. Serge Klarsfeld et Maxime Steinberg, Mémorial de la Déportation des Juifs de Belgique, Bruxelles, édité par l'Union des Déportés Juifs en Belgique et Filles et Fils de la Déportation, 1982.
} 
le convoi X, transférés de ce camp le 15-9-1942 vers les camps d'extermination de Haute Silésie ». Cette dernière liste aligne 516 noms de Juifs arrêtés le 11 septembre 1942 dans le nord de la France. Ces listes ont été dressées, rapidement, après la guerre ce qui explique l'imprécision de la destination. À partir de ces documents, Thierry Kleiman et moimême avons réussi à publier la liste des personnes raflées, le 11 septembre 1942 et déportées vers Auschwitz par le convoi X qui partit de la caserne Dossin de Malines le 15 septembre $1942^{14}$. Toujours à partir de ces listes d'archives, j'ai pu dresser la liste des convois ayant quitté Malines avec des personnes arrêtées dans le nord de la France, un autre jour que le 11 septembre 1942, et déportées dans une dizaine de convois, ainsi que la liste de Juifs arrêtés mais libérés de la caserne Dossin ${ }^{15}$.

D'autres fonds d'archives peuvent être mis à profit pour connaître le sort de ces travailleurs et de leur famille : les archives départementales du Nord et du Pas-de-Calais renferment de nombreux dossiers sur les Juifs durant la guerre, les archives municipales de Lille sont aussi utiles ${ }^{16}$.

Confrontant tous ces matériaux d'archives entre eux et avec des témoignages de survivants ou descendants de survivants, il est possible de reconstituer les familles et de connaître leur sort pour quelques-unes d'entre elles, vouées à l'extermination. Plusieurs membres de ces familles furent raflés le 11 septembre 1942 et déportés vers Auschwitz par Malines, en l'absence des pères de famille qui, eux, se trouvaient au chantier de Champaubert-aux-Bois. S'ils rentrèrent à leur domicile, après avoir déserté le chantier, ce fut pour constater la disparition de leurs proches. D'autres ont eu la chance de retrouver les leurs mais confinés dans la clandestinité.

Dans l'énumération qui suit, je présente la famille telle qu'elle s'est déclarée pour le recensement de 1942 : femme et enfants avec les métiers ou occupations, les dates et lieux de naissance (il y a parfois de petites différences avec les renseignements livrés par les fiches du chantier de Champaubert-aux-Bois, j'ai donc souligné ces différences, sauf pour l'orthographe des noms de lieux polonais). Puis, quand c'est possible, je

\footnotetext{
${ }^{14}$ Danielle Delmaire, Thierry Kleiman, « Liste des déportés du convoi X » Tsafon, revue d'études juives $d u$ Nord, $\mathrm{n}^{\circ}$ 9-10, été-automne 1992, p. 38-66, avec présentation et commentaire.

${ }^{15}$ Danielle Delmaire, «Liste des convois de la déportation partis de Malines, dans lesquels se trouvent des Juifs arrêtés dans le Nord » Tsafon, revue d'études juives du Nord, $\mathrm{n}^{\circ} 21$, printemps 1995 , p. 3-35.

${ }^{16} \mathrm{Je}$ dois remercier tout particulièrement Monique Heddebaut qui m'a communiqué sans réticence tous les dossiers qu'elle a compulsés dans ces différents centres d'archives.
} 
précise ce qui est advenu à chacun des membres des familles. Sauf indication contraire, les personnes sont toutes nées en Pologne et à côté du nom de famille figure l'adresse d'habitation qui est toujours à Lille sauf pour Liberman Jech.

\section{BLANK : 133 rue Gustave Delory}

Sÿa né le 20/2/1895 à Zgierzi, manœuvre, Polonais, 3 enfants, Jeta Rosa, Maurice, Samuel.

Rayzla Malka née Fuks le 15/3/1905 à Tyszowice, Polonaise.

Le couple est marié en 1930 et de cette union sont nés trois enfants.

Jeta Rosa née le 8/1/1931 à Lille, écolière, naturalisée le 26/2/1931.

Maurice Hasard né le 5/10/1933 à Lille, écolier, naturalisé le 19/3/1934.

Samuel est né en $1940^{17}$.

Rayzla Blank a été déportée par le convoi X, $\mathrm{n}^{\circ} 222^{18}$.

Lors de la rafle du 11 septembre 1942, les trois enfants ont été sortis de la gare de Lille-Fives par le cheminot Marcel Hoffmann ${ }^{19}$. Quant à Maurice, il fait partie des enfants sauvés de la rafle et confiés par Léon Leser à l'abbé Stahl qui cacha des garçons juifs dans son patronage, au Buisson dans la banlieue proche de Lille. En octobre 1942, les enfants sont placés dans un autre établissement à Bouvines où ils souffrent de la faim. Cachés jusqu'à la fin de la guerre, ils survivent ${ }^{20}$.

\footnotetext{
17 Témoignage de Maurice Blank dans le Bulletin de l'Association cultuelle de Lille, mai 2004.

${ }^{18}$ Tsafon, revue d'études juives du Nord, « 1942, L'année tragique des Juifs du Nord », n 9-10, été-automne 1992, p. 46.

${ }^{19}$ AD 59, 170 W 71 073. Dossier pour l'obtention de la Médaille de la Reconnaissance française, établi le 15 mars 1947, dans lequel Marcel Hoffmann cite les personnes qu'il a réussi à sortir de la gare dont « 3 enfants de Mr Blancke». Le sauvetage des Juifs, le 11 septembre 1942, en gare de Lille-Fives alors que le train est en partance pour le camp de rassemblement de Malines, a été étudié minutieusement par Monique Heddebaut, « Sans armes face à la rafle du 11 septembre 1942, dans la Zone rattachée à Bruxelles », Tsafon, revue d'études juives $d u$ Nord, $\mathrm{n}^{\circ}$ 70, automne 2015-hiver 2016, p. 119-168. Peu de temps après, un autre ouvrage moins solide et parfois fantaisiste est paru : Grégory Célerse, Sauvons les enfants, Lille, Les Lumières de Lille, septembre 2016.

${ }^{20}$ Des enfants raflés le 11 septembre 1942 et cachés par l'abbé Stahl ont témoigné. Leur récit est paru: Danielle Delmaire, «1942, L'année tragique des Juifs du Nord», «Témoignages et récit», Tsafon, revue d'études juives du Nord, n 9-10, été-automne 1992, p. 17-26. Par la suite, un témoignage de Maurice Blank, paru dans le Bulletin de l'Association cultuelle de Lille, mai 2004, rappelle comment il fut sorti de la gare de Fives par un cheminot et comment il a rejoint son domicile où un voisin l'a caché en attendant un ami de ses parents chez qui il a retrouvé sa sœur. Seule la maman est restée en gare et a été déportée. Comme Renée Strauss, née Grudki, il évoque l'absence de son père, lors de la rafle car « il se trouvait dans les chantiers forestiers ardennais, pour plusieurs raisons, éviter les rafles (celle du Vel d'Hiv venait d'avoir lieu) et gagner un peu d'argent
} 
Chya Blank exerçait son commerce depuis 1924, spolié il entreprend les démarches pour une réouverture de son magasin en décembre194421.

\section{BLIBAUM : 9 rue Doudin}

Lajbus né le 8/7/1909 à Tamaszaz, sans profession, célibataire, interné le 11/5/1943 à Malines et libéré le 4/9/1944 (à la Libération)22.

En général c'était le sort réservé aux Juifs qui avaient épousé une nonJuive.

BOCHNER : 35 rue Lottin

Mendel né le 15/11/1901 à Jadlowa, sans profession, Polonais, 1 enfant, Herman.

Rajza née Rubel le 21/9/1910 à Brzostek, Polonaise.

Herman né le 12/10/1934 à Lille, écolier, Français naturalisé en décembre 1934.

Mendel Bochner avait ouvert un commerce en 1931 et fut radié en septembre $1941^{23}$.

La famille Bochner n'est pas déportée, Henri-Herman Bochner fait partie des enfants secourus par l'abbé Stahl au Buisson et à Bouvines ${ }^{24}$. Cette famille habite encore rue Lottin à Lille dans les années 1950 dans le même immeuble que la famille Lewin selon le témoignage de Jean-Claude Lewin qui précise à propos de Mendel Bochner : «C'était un homme charmant, adorable, fort physiquement. Ce n'est pas étonnant qu'il effectuait un travail de bûcheron $»^{25}$.

BORNSTEIN : 38 rue Léonard Danel

Wolf né le 20/5/1898 à Lodz, sans profession, Polonais, sa femme est polonaise et catholique.

pour nous faire vivre ». En fait, il y a aussi confusion de sa part entre les Ardennes et la Marne mais il est vrai que Champaubert-aux-Bois se trouve non loin des Ardennes. En outre, le père espère éviter la rafle pour lui-même car nombreuses sont les familles qui pensent que seuls les hommes sont menacés d'arrestation ou de rafle. Sur l'abbé Stahl, lire Monique Heddebaut, « Robert Stahl, avocat, prêtre, résistant et l'orphelinat SaintPierre de Bouvines », Pays de Pévèle, 2017, n 81, p. 19-26.

${ }^{21}$ AD 59, $67 \mathrm{~W} 45147$ : Fichier des demandes de réouverture des commerces radiés selon la loi d'octobre 1941.

${ }^{22}$ Tsafon, revue d'études juives du Nord, $\mathrm{n}^{\circ} 21$, printemps 1995, p. 22.

23 AD 59, 67 W 45147.

${ }^{24}$ Voir note 20 et récit de David Bugajski à Monique Heddebaut, le 11 février 2016.

25 Témoignage de Jean-Claude Lewin, le 22 octobre 2020, dont les parents étaient copropriétaires de l'immeuble occupé par la famille Bochner. 
Wolf Borstein s'est engagé, en 1939, dans les Régiments de Marche des Étrangers Volontaires ${ }^{26}$.

Interné à Malines le 11/5/1943 puis à la prison Saint-Gilles à Bruxelles, le 10/8/1943 et libéré le 3/2/1944. Il doit sa non-déportation et sa libération probablement en tant que «mari d'aryenne». Son épouse tente des démarches auprès de l'évêché de Lille : le secrétaire rédige une lettre datée du 31 mai 1943 attestant qu'elle « fait des démarches pour obtenir son acte de baptême ainsi que celui de ses parents et de ses grands-parents $»{ }^{27}$. Cette lettre se trouve dans les archives de la caserne Dossin à Malines ce qui laisse comprendre qu'elle a été acheminée auprès des autorités allemandes. Si les certificats de baptême, à faire venir de Pologne, ont pu être présentés cela expliquerait la non déportation de Wolf Bornstein.

\section{EPSZTAIN : 133 rue Gustave Delory}

Moszek né le 3/4/1907 à Szarte, tailleur d'habits, Polonais, 2 enfants, Abraham né à Lille et Henri né le 19/6/1942 à Lille (cette naissance permet de dater le recensement des premiers jours de l'été 1942).

Sura née Aichenbaum en mars 1908 à Tacarow, Polonaise, 2 enfants.

Sura Epsztain a été déportée avec son fils Henri par le convoi X, respectivement sous les $n^{\circ} 220$ et $221^{28}$.

Moszk Epsztein s'est engagé dans les RMVE ${ }^{29}$.

GRUDKI : 73 rue de la Monnaie

Abraham né le 5/3/1898 à Crodesk, nationalité indéterminée, 1 enfant, Renée.

Laya née KSLER le 5/9/1889 à Stanislawczyk, 1 enfant, Renée.

Renée, née le 14/6/1931 à Lille, écolière naturalisée Française le 31/12/1935.

\footnotetext{
${ }^{26}$ Régiments de Marche des Étrangers Volontaires ou RMVE. Listes nominatives des étrangers qui se sont engagés à servir la France entre le $1^{\text {er }}$ septembre 1939 et le 25 juin 1940 (Légion étrangère, formation d'une armée nationale constituée en territoire français, dans les unités de l'armée française) pour les Ire, IIIe, Ve, VIe, VIIe, VIIIe, IXe et Xe régions militaires (lacunes), 1947. Pour le Nord et le Pas-de- Calais : VIe région militaire. https://www.memoiredeshommes.sga.defense.gouv.fr/fr/arkotheque/inventaires/ead ir consult.php?ref=FRMS FondsUEVACJEA EAD 01\&fam $=12$

${ }^{27}$ Cité dans Tsafon, revue d'études juives du Nord, ${ }^{\circ} 21$, printemps 1995, p. 25-26.

${ }^{28}$ Tsafon, revue d'études juives du Nord, «1942, L'année tragique des Juifs du Nord », n 9-10, été-automne 1992, p. 48

${ }^{29}$ Listes nominatives des étrangers qui se sont engagés à servir la France entre le 1er septembre 1939 et le 25 juin 1940.

https://www.memoiredeshommes.sga.defense.gouv.fr/fr/arkotheque/inventaires/ead_ir consult.php?ref=FRMS FondsUEVACJEA_EAD 01\&fam=12. Voir note 26.
} 
Engagé dans les $\mathrm{RMVE}^{30}$, Abraham Grudki est déporté par le convoi XIV, $\mathrm{n}^{\circ} 337$, qui a quitté Malines le 24/10/1942 ${ }^{31}$. Selon sa fille, il a été dénoncé. Ayant fui le chantier le 10 octobre, les retrouvailles avec sa femme et sa fille qui ont échappé à la rafle, n'ont pas duré longtemps. D'abord cachée chez une amie de ses parents à Orchies, Renée grâce à une institutrice, $\mathrm{M}^{\text {elle }}$ Chéreau, a pu intégrer le lycée Fénelon de Lille, en classe de $6^{\mathrm{e}}$ où elle fut cachée par $\mathrm{M}^{\text {elle }}$ Caudmont, avec Huguette Fuchs. Simone Caudmont a été reconnue Juste parmi les Nations (cf. témoignage de $\mathrm{M}^{\mathrm{me}}$ Renée Strauss née Grudki).

\section{KURCBARD : 49 rue de Tournai}

Mendel né le 22/9/1891 à Hornsk, tailleur, Polonais, 2 enfants, Georges, Français, né à Lille, Aline née à Lille.

Giza née Arman le 6/6/1904 à Siedle, Polonaise, 2 enfants Georges, Aline. Aline, née le 9/12/1931 à Paris $12^{\mathrm{e}}$, écolière, Polonaise.

Georges né le 27/9/1926 à Paris, Français naturalisé le 17/8/1929.

Giza et son fils Georges ont été déportés par le convoi $X$, respectivement $\mathrm{n}^{\circ} 604$ et $605^{32}$. Ils ne reviennent pas.

En l'absence du père, la maman et ses deux enfants furent victimes de la grande rafle du 11 septembre 1942. Aline a pu être sauvée par le cheminot Marcel Hoffmann qui parvint à la sortir de la gare de Fives-Lille alors que le train était en partance pour la caserne Dossin à Malines, avant la déportation à Auschwitz ${ }^{33}$. La fillette fut cachée à Fives, avec son père qui est parvenu à la rejoindre, chez Mme Minen : « Aline, ma cousine, sa mère et son frère Georges [furent] arrêtés rue de Tournai [...] Sa mère et son frère sont décédés en déportation. D’humbles gens de Fives-Lille, monsieur et madame Minen, l'hébergeront avec son père jusqu'à la fin de la guerre $»^{34}$.

\footnotetext{
${ }^{30}$ Idem.

${ }^{31}$ Serge Klarsfeld et Maxime Steinberg, Mémorial de la Déportation des Juifs de Belgique, op. cit.

${ }^{32}$ Tsafon, revue d'études juives du Nord, « 1942, L'année tragique des Juifs du Nord », n 9-10, été-automne 1992, p. 55.

${ }^{33}$ AD 59, 170 W 71 073. Dossier pour l'obtention de la Médaille de la Reconnaissance française, établi le 15 mars 1947, dans lequel Marcel Hoffmann cite «Melle Aline Kurbart » parmi les personnes qu'il a sauvées.

${ }^{34}$ Témoignage de Georges Komar, Le fabuleux destin d'un fils d'immigrés, édité à compte d'auteur, 2014, p. 42, ainsi que son autre récit paru dans Tsafon, revue d'études juives du Nord, $\mathrm{n}^{\circ}$ 9-10, été-automne 1992, p. 20-22. Voir aussi AD 59, 170 W 71073, dossier Hoffmann 1947 / témoignage.
} 


\section{LAUFER : 136 bd Victor Hugo}

David, né le 19/4/19/05 à Tarnobrzeg, sans profession, Polonais, célibataire.

David Laufer s'est engagé dans les RMVE ${ }^{35}$. Son entreprise a été administrée par un syndic liquidateur ${ }^{36}$.

LIBERMAN : 300 av. Jean Jaurès, Ronchin

Icek né le 26/5/1897 à Raintszyce, propriétaire commerçant, Polonais, 1 enfant adopté, Simone.

Gutha née Zilberberg (ou Zilberfeld) le 11/2/1901 à Wolbrom, commerçante, Polonaise, 1 enfant adopté, Simone.

Swybel Simone née le 8/3/1934, écolière, Polonaise. Elle est juive également mais sa fiche ne précise pas le lien de parenté avec ses adoptants. Icek ou Isaac Liberman avait ouvert un magasin en 1925, il fut radié en septembre 1941, il fit une demande de réouverture en janvier $1945^{37}$.

Son nom figure, avec son adresse, sur une liste d'otages qui devront " prendre place, jusqu'à Courtrai, dans le train de permissionnaires S.F. 217 » établie par le service des « affaires allemandes » du cabinet du préfet du Nord, datée du 6 janvier 1944, et adressée au chef de la «Kreis \& Standortkommandantur» de la Feldkommandantur 678 de Lille. Icek Liberman ne peut pas répondre à la convocation car il est parti début 1942 et « travaillerait pour l'autorité allemande ${ }^{38}$. En fait, dès novembre 1942, il ne travaille déjà plus au chantier de Champaubert-aux-Bois et apparemment sa trace est perdue.

MAJER : 18 rue des Tanneurs

Mendel né le 10/101891 à Sanok, sans profession, Polonais, 3 enfants, Joseph, Frida, Jacques (Français).

Mina née Landau le 24/9/1896 à Bukla, ménagère, Polonaise, 3 enfants, Joseph, Frida, Jacques (Français).

Joseph né le 1/2/1924 à Sanok, Polonais.

Frida née le 28/7/1925 à Sanok, Polonaise.

Jacques né le 11/8/1934 à Lille naturalisé Français le 5/12/1934.

\footnotetext{
${ }^{35}$ Listes nominatives des étrangers qui se sont engagés à servir la France entre le 1er septembre 1939 et le 25 juin 1940.

https://www.memoiredeshommes.sga.defense.gouv.fr/fr/arkotheque/inventaires/ead ir consult.php?ref=FRMS FondsUEVACJEA_EAD 01\&fam=12. Voir note 26.

${ }^{36}$ Archives privées d'Olivier Spriet qui les a aimablement mises à ma disposition.

${ }^{37}$ AD 59, $67 \mathrm{~W} 45147$.

${ }^{38}$ AD 59, 1 W 1847.
} 
Mina Majer et ses deux enfants Frida et Jacques sont déportés par le convoi $\mathrm{X}$, respectivement les $\mathrm{n}^{\circ} 784,785,786^{39}$.

Mendel et son fils Joseph, âgé de 18 ans, se sont donc adressés au bureau de placement pour travailler au chantier de Champaubert-aux-Bois et sont arrivés ensemble le 13 août 1942. En leur absence, le reste de la famille a été raflé et déporté. S'il revient à Lille après avoir quitté le chantier le 24 décembre 1942, Mendel ne trouve personne.

Il avait un commerce de linge et tissus et il cessa toute activité en mars $1942^{40}$.

\section{MUZNER : 6 terrasse Sainte Catherine}

Pinkas né le 3/6/1897 à Czuder, garçon de café, veuf, Polonais.

Pinkas Muzner s'est engagé dans les RMVE ${ }^{41}$.

Son nom figure aussi sur la liste d'otages qui doivent accompagner les permissionnaires allemands en janvier-février 1944 (voir Liberman Icek). L'adresse de Pinkas Muzner, qui est alors 6, terrasse Sainte-Catherine, est accompagnée de la précision suivante : "parti, il y a deux ans sans laisser d'adresse $»^{42}$. Ce qui laisse comprendre qu'une fois sorti du chantier de Champaubert-aux-Bois, il n'a pas rejoint son domicile ou, s'il est revenu à Lille, il vit caché.

Quant à son adresse rue Frédéric Mottez qu'il a donnée à l'administration du chantier, il est très probable, puisqu'il est garçon de café, que ce soit celle du café de Clara Campéas qui était un des établissements réservés aux Juifs ${ }^{43}$.

POZNANSKI : 50 rue de Poids

Szeul né le 6/90/1896 à Ruda Guzowska, sans profession, Polonais, 2 enfants Michel (Lille), Sophie (Française).

Hana née Wajsbaum en 1893 à Zyardow, Polonaise, 2 enfants Michel (Lille), Sophie (Française).

Michel né le 28/9/1925 à Zyardow, tailleur d'habits, célibataire, Polonais.

${ }^{39}$ Tsafon, revue d'études juives du Nord, « 1942, L'année tragique des Juifs du Nord », n 9-10, été-automne 1992, p. 57.

${ }^{40}$ AD 59, $67 \mathrm{~W} 45147$.

${ }^{41}$ Listes nominatives des étrangers qui se sont engagés à servir la France entre le 1er septembre 1939 et le 25 juin 1940.

https://www.memoiredeshommes.sga.defense.gouv.fr/fr/arkotheque/inventaires/ead ir consult.php?ref=FRMS FondsUEVACJEA EAD 01\&fam=12. Voir note 26.

${ }^{42}$ AD 59, $1 \mathrm{~W} 1847$.

${ }^{43}$ Témoignage de Clara Campéas que j'ai recueilli les 10 et 15 février puis le $1^{\mathrm{er}}$ mars 2021, à Lille. 
Sophie née le 6/12/1928 à Lille, célibataire Française naturalisée le 31/3/1930.

Hana et son fils Michel sont déportés par le convoi X, respectivement les $\mathrm{n}^{\circ} 724$ et $725^{44}$.

Poznanski s'est engagé dans les RMVE ${ }^{45}$.

\section{RAPOPORT : 26 rue Saint-Sauveur}

Jacques né le 19/1/1899 à Bedzin, culottier (confectionne des pantalons), Polonais, 2 enfants Français Joseph et Fanny.

Cyrla Laja née Bergman, date et lieu de naissance non renseignés, Polonaise, 2 enfants Français Joseph et Fanny.

Joseph né le 2/7/1927 à Lille, apprenti tailleur, célibataire, Français naturalisé le 10/12/1928.

Fanny Estelle née le 4/12/1935 à Lille, écolière, Française naturalisée le 15/12/1938.

Jacques Rapoport s'était engagé dans un RMVE dès $1939^{46}$.

Cyrla et son fils Joseph sont déportés par le convoi X, respectivement les $n^{\circ} 726$ et $727^{47}$.

Joseph avait été scolarisé, dans les années 1930, à l'école primaire Michelet, rue Fabricy à Lille, selon le registre tenu par la direction ${ }^{48}$.

Fanny a pu être sortie de la gare de Fives grâce à un cheminot qui l'amena chez des voisins $\mathrm{M}$ et $\mathrm{M}^{\mathrm{me}}$ Raynal qui l'ont cachée jusqu'à la fin de la guerre. Son père « qui était dans un camp de travail » pendant la rafle a pu la rejoindre et fut également caché par les Raynal ${ }^{49}$.

Jacques Rapoport avait un frère Nathan. Ce dernier s'était réfugié, dans le Lot, avec sa famille qui ne fut pas arrêtée ${ }^{50}$.

\footnotetext{
${ }^{44}$ Tsafon, revue d'études juives du Nord, «1942, L'année tragique des Juifs du Nord », n 9-10, été-automne 1992, p. 60.

${ }^{45}$ Listes nominatives des étrangers qui se sont engagés à servir la France entre le 1er septembre 1939 et le 25 juin 1940.

https://www.memoiredeshommes.sga.defense.gouv.fr/fr/arkotheque/inventaires/ead ir consult.php?ref=FRMS FondsUEVACJEA_EAD 01\&fam=12. Voir note 26.

46 Idem.

${ }^{47}$ Tsafon, revue d'études juives du Nord, « 1942, L'année tragique des Juifs du Nord », n ${ }^{\circ}$ 9-10, été-automne 1992, p. 60.

${ }^{48}$ Je dois cette information à mon collègue Jean-Baptiste Gardon, qu'il en soit remercié ainsi que l'actuelle directrice de l'école qui l'a autorisé à consulter ces registres.

${ }^{49}$ Témoignage de Fanny Rapoport dans le Bulletin de l'Association cultuelle de Lille, mai 2004 et voir le site Yad Vashem au nom de Raynal, le couple fut reconnu Juste parmi les Nations en 2001 et la cérémonie eut lieu en 2003.

${ }^{50}$ Témoignage de Jacqueline Alcaud, fille de Nathan Rapoport, le 18 octobre 2020.
} 
ROCHMAN : 4 rue des Augustins

Jacob né le 13/5/1904 à Varsovie, sans profession, Polonais, 1 enfant Thérèse Française.

Syme Rachel née Krebs le 6/7/ 1909 à Oleszyce Polonaise, 1 enfant Thérèse Française.

Thérèse née 19/7/1934 à Douai, écolière, Française naturalisée le 18/10/1938.

Jacob est déporté par le convoi XXII A, $\mathrm{n}^{\circ} 473$, parti de Malines le 20/9/1943. D'après la liste de ce convoi, il serait né le 30/5/1904 ${ }^{51}$. « Le convoi XXII A est un transport ordinaire composé de 639 étrangers dont 89 enfants. 8 déportés s'évaderont avant le passage de la frontière. Les déportés de ce transport ont été arrêtés du 27 juillet au 15 septembre 1943, [le convoi arrive à Auschwitz le 22 septembre, il y] aura 32 survivants en $1945 »^{52}$.

STULZAFT : 49 rue de Tournai

Berich, né le 6/4/1896 à Vilna (Russie), ouvrier agricole, russe réfugié, 2 enfants Oscar Jacob né à Lille Français et Jean Fernand (pas de fiche pour ce dernier dans le recensement de 1942).

Hélène née Libermann le 10/3/1914 à Ponesicz (Lituanie), Lituanienne, 2 enfants Oscar Jacob né à Lille Français et Jean Fernand.

Oscar né le 3/3/1932 à Lille, Français naturalisé le 25/5/1932.

Hélène est sauvée en gare de Fives avec ses deux enfants, Jean est né en $1940^{53}$.

Avant de s'engager pour des travaux de bûcheronnage au chantier de Champaubert-aux-Bois, Berich Stulzaft a été arrêté et interné à la forteresse de Huy en Belgique, le 21 août 1941, sans doute parce qu'il était de nationalité russe et/ou soupçonné d'être communiste. À l'été 1941, de nombreux communistes furent arrêtés, dans le Nord, et conduits à Huy, certains ont été libérés, c'est le cas de Berich Stulzaft qui sortit de Huy le 3 janvier 1942, d'autres furent déportés voire fusillés ${ }^{54}$.

\footnotetext{
${ }^{51}$ Tsafon, revue d'études juives du Nord, n 21, printemps 1995, p. 16.

${ }^{52}$ Serge Klarsfeld et Maxime Steinberg, Mémorial de la Déportation des Juifs de Belgique, op. cit., et voir Tsafon, revue d'études juives $d u$ Nord, $\mathrm{n}^{\circ} 21$, printemps 1995, p. 16.

${ }^{53}$ AD 59, 170 W 71073 : dossier Hoffmann 1947 / témoignage.

54 Livre mémorial du site de la Fondation pour la Mémoire de la Déportation http://www.bddm.org/liv/details.php?id=I.12. La citadelle de Huy fut utilisée par les Allemands comme centre d'internement pour prisonniers politiques (résistants, communistes) dont de nombreux Russes.
} 
Son commerce ayant cessé en septembre $1941^{55}$, immédiatement après son arrestation, Berich Stulzaft se trouvait sans ressource, à sa sortie de Huy. Il avait alors, sans doute, espéré gagner quelque argent et mettre sa famille à l'abri en acceptant de s'enrôler au chantier de Champaubert-aux-Bois. Après ou avant son départ, ses deux enfants ont été confiés à une nourrice à Thumesnil dans la banlieue lilloise. Arrêtée le 11 septembre 1942, la maman a dû livrer l'adresse de la nourrice où les Allemands sont allés les chercher comme le rapporte le commissaire central de police, dès le lendemain de la rafle : "J'ai l'honneur de vous rendre compte que la Feldgendarmerie allemande est allée chercher hier [à] Thumesnil [où] ils étaient en nourrice, les jeunes juifs Stubcaf Oscar, âgé de 10 ans et son frère Jean, âgé de 21 mois. Ces enfants auraient été emmenés pour être rassemblés avec leur famille qui aurait été arrêtée hier à Lille ${ }^{56}$.

Finalement, la maman, qui s'est adressée au cheminot Marcel Hoffmann, a pu être exfiltrée avec ses enfants de la gare de Fives ${ }^{57}$. Les enfants ont ensuite trouvé un refuge chez une famille fivoise : les Devos, victime d'un bombardement le 8 novembre suivant. Jacques (Oscar) Stulzaft fut hospitalisé et de nouveau Marcel Hoffmann le sauva en le sortant de l'hôpital où une infirmière entreprenait de le dénoncer à la police ${ }^{58}$.

WILNER : 73 rue de la Monnaie

Louis né le 4/12/1924 à Lille, manœuvre, célibataire, nationalité indéterminée.

Il est le cousin de Renée Strauss, née Grudki. Il réside au même domicile qu'elle et ses parents. De retour à Lille, il est sauvé par le réseau du pasteur Nick (lire le témoignage de $\mathrm{M}^{\mathrm{me}}$ Strauss, née Grudki).

ZYLBERBERG : 11 rue d'Amiens

Lejbus né le 7/8/1899 à Théciny, sans profession, Polonais.

Rywka née Marcyk le 1/6/1897 à Palajnic, ménagère, Polonaise.

\footnotetext{
${ }^{55}$ AD 59, 67 W 45147.

${ }^{56} \mathrm{AD} 59,1 \mathrm{~W}$ 1844. « Rapport du commissaire de central de police à Monsieur le Préfet régional de Lille », c'est-à-dire le préfet Carles. Le rapport est daté du 12 septembre 1942. Malgré l'orthographe fautive du nom de famille des enfants, il n'y a aucun doute sur leur appartenance à la famille Stulzaft au vu des prénoms et des âges, Oscar était aussi prénommé Jacques. J'ai délibérément occulté le nom et l'adresse de la nourrice qui a témoigné anonymement.

${ }^{57}$ AD 59, 170 W 71 073. Dossier Hoffmann 1947 / Témoignage.

58 Témoignage de Jacques Stulzaft, paru dans Le Parisien, 27 novembre 2021, à l'occasion de la remise du titre de Juste parmi les Nations au cheminot Marcel Hoffmann.
} 
Lejbus Zylberberg s'est engagé dans les RMVE ${ }^{59}$.

Son entreprise a été administrée par un syndic liquidateur ${ }^{60}$.

\section{Sort des dix-sept familles des travailleurs et le Comité de sauvetage des Juifs}

Dix-huit fiches de travailleurs juifs de Lille au chantier de Champaubert-aux-Bois sont conservées aux archives départementales de la Marne mais deux d'entre eux sont père et fils donc dix-sept familles sont dénombrées.

- six d'entre elles sont touchées par la rafle du 11 septembre 1942, en l'absence du chef de famille, l'épouse seule (Blank, Epsztain) ou l'épouse avec un ou deux enfants (Kurcbard, Majer, Poznanski, Rapoport). S'ils rentrent chez eux, après avoir déserté leur chantier entre le 10 octobre et le 24 décembre 1942, ces maris ne peuvent que constater la disparition d'une partie de leur famille.

- deux d'entre elles sont touchées par une autre déportation. C'est le travailleur lui-même qui est arrêté et déporté, rapidement après son retour comme Abraham Grudki tandis que sa femme et sa fille ont réussi à échapper à la rafle, moins rapidement pour Jacob Rochman qui est déporté un an plus tard (20 septembre 1943).

- deux d'entre elles sont touchées par l'arrestation du travailleur qui est interné mais libéré. Blibaum est interné le 11 mai 1943 mais non déporté et libéré à la Libération le 4 septembre 1944, tandis que Wolf Bornstein est interné le 11 mai 1943 également, non déporté et libéré le 3 février 1944 car son épouse est catholique donc « aryenne ».

- une famille est épargnée par la déportation : Stulzaft, bien que victime de la rafle du 11 septembre 1942, elle est sortie de la gare de Fives.

- six familles semblent être entrées dans une clandestinité efficace dans la mesure où les archives sont muettes à leur égard, au moins dans l'état actuel de nos recherches.

Au lendemain de la grande rafle du 11 septembre 1942, Léon Leser, avec l'aide d'autres personnes juives et non juives, mit sur pied un comité de sauvetage des Juifs. Après la guerre, le 4 février 1946, les membres survivants de ce comité rédigent une attestation qui certifie l'engagement

\footnotetext{
${ }^{59}$ Listes nominatives des étrangers qui se sont engagés à servir la France entre le 1er septembre 1939 et le 25 juin 1940.

https://www.memoiredeshommes.sga.defense.gouv.fr/fr/arkotheque/inventaires/ead_ir consult.php?ref=FRMS_FondsUEVACJEA_EAD_01\&fam=12. Voir note 26.

${ }^{60}$ Archives privées d'Olivier Spriet.
} 
du résistant René Douce qui était le président du «Comité clandestin d'Aide aux Juifs recherchés par la Gestapo de la Région Lilloise, en 1942, jusqu'à son arrestation »; celle-ci eut lieu en décembre de la même année pour activités communistes ${ }^{61}$. Parmi les premiers membres du Comité, ce document cite : «Messieurs Saint Maxent Louis, Smekens Paul (mort en déportation), Raby (mort en déportation), Prechner Simon, Leser Léon, Douce René ». Par la suite, d'autres personnes s'activèrent au sein du Comité dont Madame Carles, née Lang, l'épouse du préfet qui était d'origine juive, ainsi que la femme de l'inspecteur d'académie de Lille, Madame Signoret, Monsieur Tricoteux dont la position de chef du service de ravitaillement en mairie de Lille était très précieuse, le pasteur Nick de Fives, qui hébergea plusieurs Juifs avant de les disperser dans diverses familles du Cambrésis avec l'aide de son fils médecin, et le chef de gare principal de Fives-Lille, Jean Mabille, qui couvrit les actions de ses subordonnés cheminots.

Non seulement, la plupart de ces hommes avaient participé au sauvetage des Juifs en gare de Fives-Lille, mais ils avaient fourni des cachettes aux enfants et aux adultes, ainsi que de faux papiers, des cartes de ravitaillement et parvenaient à payer la pension d'enfants placés chez des « logeurs ».

Alors qu'au moment de l'arrestation de René Douce, « le Comité s'occupait déjà de 54 personnes sans compter les passagers », finalement c'est 167 Juifs adultes et enfants qu'il parvint à secourir. Léon Leser en établit la liste ${ }^{62}$ sur laquelle on peut lire les noms de quelques familles des travailleurs du chantier de Champaubert-aux-Bois: Bochner (2), Rappaport (2), Poznanski (2), Epstein (2), Kurcbard (4), Liberman (4), Maurice Blank (2), Stulzaft (4), David Laufer (1). Une fois rentrés à Lille, ces hommes se sont cachés avec les membres de leur famille qui n'ont pas été raflés.

Par leur enrôlement dans le chantier de Champaubert-aux-Bois, à l'été 1942, ces hommes ont alors pu éviter la rafle. Certains retrouvent les leurs, d'autres non.

\footnotetext{
${ }^{61}$ Cette attestation se trouve aux AD 59, $170 \mathrm{~W} 71072$ et porte la signature de Simon Prechner, Léon Leser, la veuve de Paul Smekens et Louis Saint-Maxent. On peut se référer également au travail de Monique Heddebaut, «La spécificité de la Zone dite " rattachée à Bruxelles », Tsafon, revue d'études juives du Nord, $\mathrm{n}^{\circ} 79$, printemps - été 2020, p. 17-38, notamment les p. 23-31.

${ }^{62}$ Archives privées d'Edgard Leser, fils de Léon Leser, lui-même caché durant la guerre. Liste manuscrite.
} 


\section{Conclusions}

La loyauté envers la patrie d'adoption.

Bien qu'immigrés, et parfois récemment, huit de ces travailleurs se sont engagés dans les RMVE, à l'instar d'un grand nombre de leurs coreligionnaires. Dès la déclaration de la guerre, les chefs de famille se présentent auprès des instances militaires pour participer à la défense de la France, espérant que la promesse de naturalisation sera respectée. Cette loyauté les incite parfois à respecter la législation même lorsqu'elle les discrimine et ils acceptent d'être recensés en tant que Juif, avec leur famille. Cette loyauté s'avèrera être un piège mais ils ne peuvent concevoir l'antisémitisme et la perversité d'une France qui les a accueillis.

La précocité de certaines arrestations.

Dès le mois d'août 1941, Berich Stulzaft est interné à la forteresse de Huy, en Belgique. Il n'est pas arrêté en tant que Juif mais sa nationalité russe le rend suspect de communisme et il est bien repéré comme Juif. Des soupçons d'appartenance au judéo-bolchevisme pèsent sur lui.

Les moyens de survie.

Spoliés donc sans ressources, les chefs de famille saisissent toutes les occasions pour faire vivre les leurs. Ils font confiance aux propositions du Commissariat de lutte contre le chômage et s'enrôlent dans les chantiers qui fonctionnent pour les Allemands mais qui sont placés sous la surveillance de la gendarmerie française. En réalité, le chômage leur a été imposé par la spoliation de leurs biens! Le chantier serait donc une solution pour mettre fin à leur désœuvrement forcé. Les Juifs de Belgique ont été soumis à cette même sorte de chantage : spoliés et sans revenu, ils ont été convoqués pour travailler dans les camps du Boulonnais pour l'Organisation Todt. Le même procédé de recrutement fut utilisé pour envoyer des Juifs anversois travailler au Judenlager des Mazures. Ces embauches se sont révélées être des leurres puisque ces travailleurs furent déportés vers Auschwitz en octobre 1942. À ce moment, les Juifs lillois du chantier de Champaubert-aux-Bois comprennent qu'ils ont été bernés et mesurent le danger : ils préfèrent s'évader du chantier.

La nécessaire résistance.

Évadés et donc en danger, d'autant que leur domicile est connu, ces hommes pour la plupart sont rentrés chez eux pour constater le désastre : 
l'épouse et les enfants ont été déportés, sinon ils se cachent. Ils leur restent à se cacher eux aussi, ce que font Mendel Kurcbard et Jacques Rapoport qui retrouvent leur enfant dans sa cachette.

L'évasion et la cachette sont des formes de résistance. La décision est prise en conscience des dangers mais le rejet de la persécution est plus fort. $\mathrm{Ne}$ plus travailler pour les Allemands et leurs complices du Gouvernement de Vichy qui ont ouvert les chantiers, se cacher pour empêcher la pleine réalisation de la " solution finale du problème juif », même si celle-ci n'est pas clairement perçue, sont autant de décisions qui enrayent la machine de production pour l'effort de guerre allemand et celle de l'extermination des Juifs. Bien que non armée et non violente, cette forme de résistance s'oppose à l'ordre de guerre allemand et vichyste.

Enfin la création du Comité de sauvetage est aussi une forme de résistance et des résistants politiques, Douce et Saint Maxent, en font partie.

Il faut également insister sur la prise en charge de leur sort par les Juifs eux-mêmes. Certes, en certaines occasions, l'aide de non-Juifs est indispensable pour être cachés et ravitaillés, mais les Juifs décident d'euxmêmes de s'évader, de se cacher et finalement de s'organiser en un comité de secours. Ils sont victimes de la persécution raciale mais ils ne s'y soumettent pas et s'efforcent de la contrecarrer. Cette étude montre que «l'ère victimaire » est révolue ${ }^{63}$.

Cet épisode de la micro histoire ne s'inscrit-il pas dans l'Histoire des Juifs? Nombreuses furent les persécutions subies par les Juifs en tout temps et en tout lieu. Souvent la parade fut de fuir d'où les diasporas comme celle des Séfarades du Bassin méditerranéen après leur expulsion de la péninsule ibérique, ou bien de se cacher comme les marranes de cette même région. Mais la parade fut aussi de décider de créer par eux-mêmes un État indépendant en développant l'idéologie sioniste.

Les sources d'archives sont abondantes et il est possible que des documents aient pu m'échapper, notamment en ce qui concerne les spoliations. C'est pourquoi ce travail pourrait être complété, à l'avenir.

\footnotetext{
${ }^{63}$ L'origine de l'expression vient de l'historien Iannis Roder auteur de Sortir de l'ère victimaire. Pour une nouvelle approche de la Shoah et des crimes de masse, Paris, éditions Odile Jacob, 2020. Quant à l'expression « sans armes face à ... », elle est utilisée par Jacques Sémelin, Sans armes face à Hitler : 1939-1945, la résistance civile en Europe, Paris, les Arènes, 2013, qui complète un ouvrage édité en 1989 et réédité en 1998. Du même, voir aussi La survie des Juifs en France : 1940-1944, Paris, CNRS éditions, 2018. Monique Heddebaut s'inscrit dans cette même analyse de la résistance sans armes et de la fin de l'ère victimaire dans son étude " Sans armes face à la rafle du 11 septembre $1942 »$, Tsafon, revue d'études juives du Nord, n 70 , art.cité.
} 


\section{TEMOIGNAGE DE MADAME RENÉE STRAUSS Neuilly, 14 avril 2008}

En 1941, mon père apprit que les Juifs pouvaient aller travailler dans les Ardennes. Comme il ne pouvait plus exercer dans son magasin, il a accepté et il a travaillé comme bûcheron environ six mois dans les Ardennes. Mais les conditions de travail étaient très pénibles et il a préféré quitter pour revenir à Lille. Il a alors pensé partir pour la Suisse mais le passeur demandait $5000 \mathrm{~F}$ par personne, or la famille se composait de papa, maman, mon cousin Louis et moi. C'était trop cher et par la suite nous avons appris que ce passeur empochait l'argent mais livrait les Juifs aux Allemands [...]

Nous avons échappé à la rafle du 11 septembre 1942 car nous n'avons pas entendu frapper à $6 \mathrm{~h}$ du matin et apparemment on n'a pas insisté. Le matin, maman est partie ; je suis sortie vers 9 ou $10 \mathrm{~h}$ pour la rejoindre en empruntant le couloir qui longeait le magasin et à ce même moment des soldats allemands sont entrés dans le magasin. J'ai couru pour retrouver maman. Elle a enlevé mon étoile et m'a fait prendre un train pour me rendre à Orchies chez ma nourrice. Mon père était absent et je ne sais plus où il se trouvait. Mon cousin Louis Wilder a pu trouver refuge chez le pasteur Nick qui l'a caché grâce à son réseau.

Je suis restée quelques semaines à Orchies, ma mère se cachait à Nomain. Mes parents sont venus me rendre visite à Orchies. C'est la dernière fois que j'ai vu mon père. Je me souviens que nous étions dans une petite maison près de la gare et des établissements de la chicorée Leroux. Nous étions chez des amis et nous nous blottissions l'un contre l'autre tant nous avions froid. Je me souviens bien de ces instants [...].

Par la suite mon père a été arrêté sur dénonciation au début d'octobre 1942. Il est parvenu à s'échapper de la Kommandantur mais il a été vite repris par des miliciens alors qu'il errait dans la rue. Il a été déporté à Auschwitz, de Malines, en octobre $1942^{64}$. La nuit de son arrestation, je me suis réveillée en hurlant.

\footnotetext{
${ }^{64}$ Voir Maxime Steinberg et Serge Klarsfeld, Le Mémorial de la Déportation des Juifs de Belgique, op. cit., qui donne le nom de Grudki Abram né le 3.5.98, déporté par le convoi XIV et portant le $n^{\circ} 337$. Selon la même source, le convoi XIV emportait 995 personnes. C'est un des convois qui, à la fin du mois d'octobre 1942, déporte les travailleurs forcés de l'Organisation Todt qui proviennent des camps du Boulonnais. Les convois XIV et XV partis tous deux de Malines le 24 octobre 1942 arrivent à Auschwitz le 26 octobre. Sur les 1472 personnes des deux convois, 576 sont immatriculées, les autres sont exterminées. De ces deux convois, 45 personnes sont encore en vie en 1945.
} 